\title{
Zur Bedeutung der Vertragsbindung im Arbeitsrecht
}

\section{Bericht über die 2. Assistententagung im Arbeitsrecht unter besonderer Berücksichtigung des Vortrags von Clemens Latzel „Arbeiten nach Wunsch“}

Alexander Stöhr*

Vom 27. bis 28. Juli 2012 fand in Mainz die 2. Assistententagung im Arbeitsrecht statt. Diese noch junge Veranstaltung will dem arbeitsrechtlichen Nachwuchs Gelegenheit zum fachlichen und persönlichen Austausch geben, da er gerade an kleineren Fachbereichen häufig auf sich alleine gestellt ist. Die Idee eines jährlichen Treffens kam auf, als im Oktober 2010 die Tagung „Arbeitsrecht und Methode“ in Köln auf große Resonanz stieß. Auch in diesem Jahr folgten über 40 Nachwuchswissenschaftler ${ }^{1}$ der Einladung von Katharina Dahm (Mainz) und Katharina Uffmann (Bayreuth) und fanden sich bei tropischen Temperaturen im Campus der Johannes GutenbergUniversität ein.

Der Nachwuchs dominierte auch den Kreis der Referenten, der sich größtenteils aus Doktoranden und Habilitanden zusammensetzte. Da die Tagung und das Call for Papers nicht unter einem Leitthema standen, wurden den Teilnehmern vielfältige Ausschnitte des Arbeitsrechts präsentiert. Den Eröffnungsvortrag hielt Sudabeh Kamanabrou (Bielefeld), die rechtsvergleichend über die Kettenbefristung zur Vertretung referierte. Es folgte ein prozessrechtlicher Vortrag von Bernhard Ulrici (Leipzig). Der Referent ging der Frage nach, inwieweit die internationale Zuständigkeit nach der Brüssel I-VO auf Verfahren nach $\$ 2$ a Abs. 1 Nr. 1 ArbGG anwendbar ist. Sodann befasste sich Stefan Greif (Bochum) mit der Unanwendbarkeit von nationalem Recht bei nicht ordnungsgemäßer Umsetzung arbeitsrechtlicher EU-Richtlinien. Diese folge nach der Rechtsprechung des EuGH nicht aus einer horizontalen unmittelbaren Richtlinienwirkung, sondern vielmehr aus dem Anwendungsvorrang unionaler Grundrechte. Den letzten Vortrag des ersten Tages hielt Volker Ströbele (Robert Bosch $\mathrm{GmbH}$ ), der als Praktiker über Mitbestimmung bei globalen Unternehmensprozessen referierte. Der zweite Tag begann mit dem Vortrag von Clemens Latzel (München) über Arbeiten nach Wunsch, der sogleich eingehender dargestellt wird. Als nächstes befasste sich Gerrit Forst (Bonn) mit dem neuen Beschäftigtendatenschutz. Es folgte ein weiterer Vortrag aus der Praxis, der von Pascal Ludwig (Greenfort) über die Kündbarkeit von GmbH-Geschäftsführern gehalten wurde. Zu guter Letzt referierte Christian Schneider (Passau) über $\$ 174$ BGB, der sich insbesondere bei Kündigungen oftmals als tückisch erweise. Dabei ging der Referent vor allem auf anderweitige Kenntnis von der Bevollmächtigung i.S.v. $\$ 174$ S. 2 BGB ein.

* RA Dr. Alexander Stöhr ist Wissenschaftlicher Mitarbeiter an der Philipps-Universität Marburg, Institut für Handels-, Wirtschafts- und Arbeitsrecht (Professor Dr. Markus Roth).

1 Aus Gründen der größeren Übersichtlichkeit und besseren Lesbarkeit wird im Folgenden auf die Nennung beider Geschlechter verzichtet. 
Im Folgenden soll der Vortrag von Clemens Latzel zu dem Thema „Arbeiten nach Wunsch" näher besprochen werden. ${ }^{2}$ Bereits der Titel ruft mannigfaltige Assoziationen hervor: Können Arbeitnehmer etwa die Arbeitszeit frei einteilen oder eine angemessene Büroausstattung mit Klimaanlage verlangen? Schon dies zeigt, dass es sich um ein übergreifendes und facettenreiches Thema handelt. Ausgangspunkt des Vortrags bildete der Grundsatz „pacta sunt servanda“, wonach Verträge kraft privatautonomer Einigung bindend sind. Im Arbeitsverhältnis als Dauerschuldverhältnissen würde eine zu starre Bindung jedoch zu unbilligen Ergebnissen führen, da sich die persönlichen Verhältnisse der Arbeitnehmer im Laufe der Zeit aus zahlreichen Gründen ändern können, man denke nur an altersbedingte Leistungsminderung oder Schwangerschaft. Um den betroffenen Arbeitnehmer nicht auf unsichere Änderungsverhandlungen zu verweisen, hat der Gesetzgeber zahlreiche Möglichkeiten zur vertragsimmanenten Anpassung geschaffen. In seltenen Fällen erfolgt dies unmittelbar durch Gesetz, namentlich im Falle eines Betriebsübergangs oder bei einem unwirksamen Arbeitnehmerüberlassungsvertrag. Diese Zurückhaltung ist auch sachgerecht, da gesetzliche Anpassungen einen gravierenden Eingriff in die (negative) Vertragsfreiheit der Parteien darstellen, der z.B. bei einem Betriebsübergang durch das Widerspruchsrecht nach $\$ 613$ a VI BGB abgefedert werden muss. In den meisten Fällen ist die Anpassung vielmehr als Gestaltungsrecht ausgestaltet und damit in das Ermessen des Arbeitnehmers gestellt.

In diesem Zusammenhang ging der Referent zunächst auf Gestaltungsrechte nach allgemeinem Zivilrecht ein. Dabei erörterte er die Streitfrage, ob bei Arbeitsverträgen eine Anpassung wegen Störung der Geschäftsgrundlage nach $\$ 313$ BGB möglich ist. Nach überwiegender Ansicht ist dieses Institut wegen Vorrangs der Kündigungsvorschriften nur subsidiär anwendbar. ${ }^{3} \mathrm{Da}$ jedoch bereits eine Änderungskündigung den Bestand des Arbeitsverhältnisses empfindlich in Frage stellt und eine Anpassung wegen Störung der Geschäftsgrundlage ohnehin nur unter strengen Voraussetzungen möglich ist, plädiert Latzel dafür, die Anwendung von $\$ 313$ BGB auch auf ungekündigte Arbeitsverhältnisse nicht per se abzulehnen. Gerade wegen der hohen An-

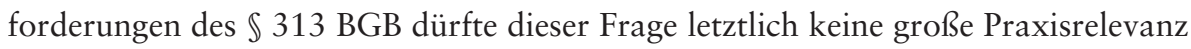
zukommen.

Sodann widmete sich der Referent den spezifisch arbeitsrechtlichen Problemfeldern. Dem Abschluss eines Arbeitsvertrages ist die Auswahl eines geeigneten Arbeitgebers vorgeschaltet. Ein Anspruch, bei dem Wunscharbeitgeber arbeiten zu können, besteht jedoch grundsätzlich nicht. Für die seit Einführung des AGG häufig auftretenden Diskriminierungsstreitigkeiten sieht $\mathbb{1 5}$ AGG lediglich Schadensersatz und

2 Die nachfolgenden Ausführungen beziehen sich auf ausgewählte Schwerpunkte des Vortrags und beruhen, soweit nicht anders gekennzeichnet, auf den persönlichen Ansichten des Verf.

3 BAG AP Nr. 60 zu $\$ 138$ BGB. 
Entschädigung vor, ein Anspruch auf Abschluss eines Arbeitsvertrags wird nach Abs. 6 ausdrücklich ausgeschlossen. Als Anspruchsgrundlage kommt ansonsten nur der im Zusammenhang mit Kontrahierungszwang geläufige $\mathbb{} 826$ BGB in Betracht. Eine vorsätzliche sittenwidrige Schädigung kann sich jedoch wegen $\$ 15$ Abs. 6 AGG nur aus anderen Gründen als einer Diskriminierung ergeben, sodass die Voraussetzungen kaum jemals erfüllt und noch seltener tatsächlich nachweisbar sein werden. Etwas anders gilt freilich im öffentlichen Dienst, wo die Besten einen Einstellungsanspruch haben, wenn sie der Stellenbeschreibung vollständig entsprechen und ihre Einstellung die einzig ermessensfehlerfreie Entscheidung ist. Diesem Anspruch könnte in der heutigen Zeit verstärkt Bedeutung zukommen, wenn die öffentliche Einstellungspraxis zurückhaltend ist und sich überdies nicht selten am gewünschten Parteibuch orientiert. ${ }^{4}$ Eine Sonderform des Einstellungsanspruchs ist der Widereinstellungsanspruch, der sich insbesondere bei Kündigungen und Betriebsübergängen aus $\mathbb{2} 242$ BGB bzw. den nachwirkenden Fürsorgepflichten des Arbeitgebers ergeben kann. Da im Kündigungsrecht das Prognoseprinzip gilt und die Wirksamkeitskontrolle nur auf den Zugangszeitpunkt bezogen ist, kann es vorkommen, dass sich die Prognose - z.B. dass der betroffene Arbeitsplatz wegfällt - nachträglich als falsch herausstellt, die Kündigung aber wirksam bleibt. Stärker als bei herkömmlichen Bewerbern ist die Rechtsstellung von Auszubildenden, denen nach Beendigung ihrer Berufsausbildung ein Übernahmeanspruch eingeräumt wird.

Weiter besprach Latzel die Arbeit in gewünschtem zeitlichem Umfang und zu Wunschzeiten. Dabei ging er zunächst auf Ansprüche nach dem TzBfG auf Reduzierung bzw. Verlängerung der Arbeitszeit gem. $\mathbb{S} 8$, 9 TzBfG ein. Es folgten Ausführungen zur Elternzeit nach BEEG, Pflegezeit nach PflegeZG, schwerbehinderungsbedingte Befreiung von Mehrarbeit ( $\$ 124$ SGB IX) sowie zu dem Umsetzungsanspruch von Nachtarbeitern aus $\$ 6$ Abs. 4 ArbZG. Nur kurz befasste sich Latzel mit Arbeiten für gewünschtes Entgelt, da es keinen gesetzlichen Anspruch auf Gehaltsanpassung gibt. Wenn Arbeitnehmer ihren Arbeitgeber nicht zu einer Gehaltserhöhung überreden können, müssen sie sich kollektiv organisieren. Auch allgemeinverbindliche Mindestlöhne, die seit Jahren ein politischer Brennpunkt sind, bewirken lediglich eine Änderung des gesetzlich geschuldeten Entgelts und keine vertragliche Modifikation. Juristisch spannender ist das Thema „Entgelt nach Wunsch“ daher aus Sicht des Arbeitgebers, der ein Interesse daran hat, auf die wirtschaftliche Situation flexibel reagieren zu können. Das entsprechende vertragliche Instrumentarium, insbesondere Widerrufs-, Freiwilligkeits- und Anrechnungsvorbehalt, unterliegt jedoch einer strengen AGB-Kontrolle. ${ }^{5}$

4 Vgl. dazu I.v. Münch, Promotion, 3. Aufl., Tübingen 2006, S. 14.

5 Dazu etwa M.Henssler/W.Moll, AGB-Kontrolle vorformulierter Arbeitsbedingungen, München2011. 
Im Zentrum des Vortrags stand schließlich die Arbeit am gewünschten Arbeitsplatz. Dabei befasste sich Latzel zunächst mit einem Anspruch auf einen „menschengerechten Arbeitsplatz“. Damit ist nicht etwa ein angenehmes Betriebsklima mit freundlichen Kollegen, fehlerlosen Vorgesetzten und moderater Arbeitsbelastung gemeint, sondern nur ein Schutz vor Gesundheits- und Persönlichkeitsbeeinträchtigungen. Einschreiten muss der Arbeitgeber daher vor allem gegen Diskriminierungen ( $\$ 12$ Abs. 3 AGG) oder gar Mobbing ( $\$ 241$ Abs. 2 BGB) durch Kollegen, wobei ihm jedoch ein Ermessensspielraum zusteht und der betroffene Arbeitnehmer keinen Anspruch auf bestimmte Maßnahmen hat. Ein Anspruch auf einen ungefährlichen und stressfreien Arbeitsplatz kann sich nur aus $\$ 618$ BGB i.V.m. öffentlich-rechtlichen Arbeitsschutznormen ergeben, womit jedoch keine Vertragsanpassung oder Zuweisung von vertragsfremden Tätigkeiten erreicht werden kann. In diesem $\mathrm{Zu}$ sammenhang eröterte Latzel den aktuellen Vorschlag der IG Metall zu einer AntiStress-Verordnung, die das ArbSchG ergänzen soll. ${ }^{6}$ Danach muss der Arbeitgeber Maßnahmen treffen, um die Arbeitsfähigkeit der Beschäftigten für die Dauer ihres Arbeitslebens zu bewahren; im Einzelnen sollen die Arbeitnehmer ihre Fähigkeiten in einem „menschengerechten “ Arbeitspensum erhalten und erweitern können, ohne qualitativ über- oder unterfordert zu werden. Latzel kritisierte jedoch, dass offen bleibe, wie Arbeitgeber dem praktisch gerecht werden sollen. ${ }^{7}$ Lebensnäher sei hingegen der Umgang mit schwangeren und stillenden Arbeitnehmerinnen ausgestaltet, was sich insbesondere an dispositiven Beschäftigungsverboten $(\mathbb{S} 3$ Abs. 2, 6 Abs. $1 \mathrm{MuSchG)}$ zeige. So können Schwangere bei Fehlen eines absoluten Beschäftigungsverbots auf ihren ausdrücklichen Wunsch auch in den letzten sechs Wochen vor der Entbindung beschäftigt werden. Dem könne der Arbeitgeber nicht widersprechen, da der Gesetzgeber Schwangere nicht bevormunden, sondern als „mündige Arbeitgeber" selbst entscheiden lassen wolle. Ist die Schwangere durch ein Beschäftigungsverbot gehindert, ihre vertragliche Arbeitsleistung zu erbringen, kann ihr der Arbeitgeber auch vertragsfremde Ersatztätigkeiten anbieten, was eine arbeitsrechtliche Besonderheit darstellt. Sofern die Ersatztätigkeit der Billigkeit entspricht, führt eine Ablehnung durch die Arbeitnehmerin zum Verlust ihres Anspruchs auf Mutterschutzlohn. ${ }^{8}$ Die bislang ungeklärte Frage, ob ein Anspruch auf Zuweisung einer billigen Ersatzbeschäftigung besteht, verneint Latzel, da das MuSchG keine dem $\$ 81$ Abs. 4 SGB IX entsprechende Regelung enthält und außerdem Schwangere durch ihren Mutterschutzlohn wirtschaftlich abgesichert seien.

6 Abrufbar unter http://www.igmetall.de/cps/rde/xbcr/internet/docs_ig_metall_xcms_188529_2.pdf, Stand: 6. August 2012.

7 Kritisch auch das Institut für angewandte Arbeitswissenschaft (ifaa) und der Berufsverband Deutscher Psychologinnen und Psychologen (BDP), dazu S.Astheimer, FAZ v. 19. Juli 2012.

8 BAG NZA 2001, S. 386. 
Sodann warf Latzel die Frage auf, ob Arbeitnehmer bei Leistungsdefiziten einen Anspruch auf einen (minder)leistungsgerechten Arbeitsplatz haben. Diese Frage betrifft ein weites, vielfach unter dem Stichwort „Low-Performer"9 diskutiertes Problemfeld. Im Hinblick auf krankheitsbedingte Leistungsdefizite ist anerkannt, dass diese auf einem leidensgerechten Arbeitsplatz weiterbeschäftigt werden müssen, der notfalls durch Ausübung des Direktionsrechts freigemacht werden muss. Auch bei sonstigen Leistungsdefiziten leitet das BAG aus $\mathbb{2 4 1}$ Abs. 2 BGB die Nebenpflicht des Arbeitgebers ab, die Voraussetzungen für die Vertragsdurchführung zu schaffen, Erfüllungshindernisse zu beseitigen und dem anderen Teil den angestrebten Leistungserfolg zukommen zu lassen. ${ }^{10}$ Bei Verstößen des Arbeitgebers verneint der Fünfte Senat jedoch einen naheliegenden Annahmeverzug. Ansonsten könne sich der Arbeitnehmer seine Aufgaben selbst bestimmen, was mit den Grundsätzen des Direktionsrechts unvereinbar sei. Jedoch mache sich der Arbeitgeber wegen Verstoßes ge-

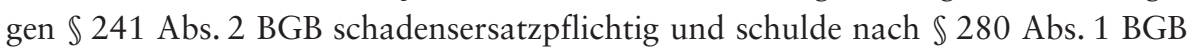
Ersatz eines eventuellen Verdienstausfalls, wenn eine anderweitige Beschäftigung möglich und zumutbar wäre. ${ }^{11}$

Diese Lösung wirkt umständlich, da es dem Arbeitnehmer in erster Linie um seinen Lohn geht, den ihm der Fünfte Senat nur über den Umweg des Schadensersatzes als Sekundärrecht zuspricht. Auch in dogmatischer Hinsicht vermag sie nicht zu überzeugen. Wenn der Arbeitgeber nämlich überhaupt keine Arbeit zuweist und der Arbeitnehmer daher nicht arbeiten kann, befindet er sich unproblematisch in Annahmeverzug ( $\$ 296$ BGB: Eröffnung einer Arbeitsmöglichkeit als Mitwirkungshandlung des Arbeitgebers). Warum etwas anderes gelten soll, wenn der Arbeitgeber eine ungeeignete Arbeit zuweist, die der Arbeitnehmer ebenfalls nicht verrichten kann, erschließt sich nicht. Damit würde auch nicht in das Direktionsrecht des Arbeitgebers eingegriffen, da die Auswahl der leistungsgerechten Arbeit immer noch bei ihm liegt. Wünsche des Arbeitnehmers sind als bloße Anregung zu verstehen und für den Arbeitgeber nur verbindlich, wenn sie die einzig mögliche Tätigkeit benennen. Außerdem bekäme die Zuweisung von leistungsgerechter Arbeit danach den Charakter einer Obliegenheit, die der Arbeitgeber zur Vermeidung von Annahmeverzug vornehmlich im eigenen Interesse zu erfüllen hätte. Dies erscheint sachgerechter als die Annahme einer echten (Neben)Pflicht, da der Arbeitnehmer sein Geld letztlich ohnehin bekommt und der Arbeitgeber durch mangelhafte Arbeitsleistung selbst benachteiligt ist. Die - im Prozess von Arbeitnehmer zu beweisenden - Voraussetzungen eines so verstandenen Annahmeverzugslohns wären also die minderleistungsbedingte Ungeeignetheit der bisher zugewiesenen Tätigkeit und das Vorhandensein zu-

9 Dieser Begriff hat bereits Einzug in die Rechtsprechung gehalten, vgl. LAG Berlin-Brandenburg NZARR 2011, S. 522 (525).

10 BAG NZA 2010, S. 1119 (1121).

11 BAG NZA 2010, S. 1119 (1120 f.). 
mindest einer möglichen und geeigneten Alternativtätigkeit. Nach der Linie des Fünften Senats sind diese Fragen in ähnlicher Form im Rahmen des Prüfungspunktes „Pflichtverletzung“ zu prüfen.

Während es bislang nur um die Umsetzung im Rahmen eines bestehenden Arbeitsverhältnisses ging, geht der Sechste Senat noch einen Schritt weiter und entnimmt \241 Abs. 2 BGB sogar einen Anspruch auf Vertragsanpassung. Danach könnten Arbeitnehmer einen Arbeitsplatz verlangen, der ihrer verbliebenen Leistungsfähigkeit und ihren Kenntnissen entspricht, beiden Parteien zumutbar und natürlich auch frei ist. ${ }^{12}$ Nicht gefordert wird hingegen, dass der Arbeitgeber einen geeigneten Arbeitsplatz erst einrichtet. In dieser Rechtsprechung sieht Latzel eine Umgehung der strengen Anforderungen, unter denen eine Vertragsanpassung nach $\$ 313$ BGB verlangt werden kann: Statt einer schwerwiegenden Änderung der Umstände die Geschäftsgrundlage geworden sind, reiche nun eine unerwartete Änderung tatsächlicher Verhältnisse aus; statt der Unzumutbarkeit des Festhaltens am unveränderten Vertrag unter Berücksichtigung aller Umstände des Einzelfalls und der vertraglichen wie gesetzlichen Risikoverteilung reiche das Drohen von erheblichen Schäden für den Arbeitnehmer als Schuldner aus; und an die Stelle der hypothetischen Überlegung, mit welchem Inhalt die Parteien den Vertag in Vorausahnung der Veränderung geschlossen hätten, trete die Vertragsanpassung nach den Wünschen des Arbeitnehmers als Schuldner. Ein solcher Anspruch könne auch nicht dadurch legitimiert werden, dass eine entsprechende Vertragsänderung wirtschaftlich beiden Seiten dient, da in der Praxis ohnehin regelmäßig Änderungsverträge auf freiwilliger Basis geschlossen werden, ohne dass es einer derartigen Nebenpflicht bedarf. Zudem werde dem Arbeitgeber die Möglichkeit genommen, neue Kräfte von außen oder Nachwuchskräfte von innen zu berücksichtigen. Ein derartiger Bestandsschutz sei gesetzlich nur bei Schwerbehinderten vorgesehen ( $\$ 81$ Abs. 4 SGB IX). Damit hat Latzel gewichtige Einwände herausgearbeitet, mit denen sich das $B A G$ zumindest auseinandersetzen sollte.

Als Fazit hält Latzel fest, dass der Grundsatz „pacta sunt servanda“ im Arbeitsrecht mehr und mehr gelockert und in ein „pacta erant servanda“ gewandelt wird. Kranken, schwerbehinderten und nun auch leistungsgeminderten Arbeitnehmern werde auf der Grundlage von Nebenpflichten und orientiert am Kriterium der Zumutbarkeit ein übersteigerter Bestandsschutz eingeräumt. Durch diese Aufweichung der Vertragstreue wüssten die Arbeitgeber bei der Einstellung immer weniger, worauf sie sich einlassen, was die Begründung von herkömmlichen Arbeitsverhältnissen unattraktiver mache. Die Begründung von Nebenpflichten nach Zumutbarkeitskriterien drohe ins Uferlose abzugleiten, da die Zumutbarkeit im Zweifel erst vom Richter bestimmt wird. Das Fazit des Verfassers zu diesem gelungenen Vortrag besteht darin, 
dass Latzel ein interessantes und vielschichtiges Thema präsentiert und dabei einige bedenkliche, wenn nicht gar falsche Entwicklungen der Rechtsprechung aufgezeigt hat. Man darf auf die eigenen Ausführungen des Referenten gespannt sein, die in dem angekündigten Tagungsband erscheinen werden. 